\title{
Targeting Antibiotic Resistant Salmonella enterica: Bio-matrix Based Selection and Bioactivity Prediction of Potential Nutraceuticals
}

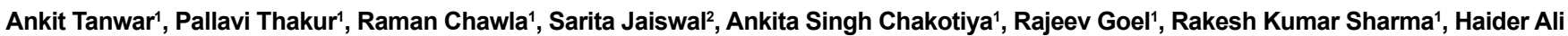

Khan $^{3}$ and Rajesh Arora ${ }^{4 *}$

${ }^{1}$ Division of CBRN Defence, Institute of Nuclear Medicine and Allied Sciences, Delhi, India

${ }^{2}$ Department of Plant Sciences, Campus Drive College of Agriculture and Bioresources University of Saskatchewan Saskatoon, Saskatchewan, Canada

${ }^{3}$ Department of Medical Elementology and Toxicology, Jamia Hamdard, Delhi, India

${ }^{4}$ Office of DG (LS), Defence Research and Development Organisation, DRDO Bhawan, Delhi, India

\begin{abstract}
Aim: Potential herbal leads as novel therapeutic alternatives against Multi-Drug Resistant Salmonella enterica serovar typhimurium were investigated and validated by mol inspiration.

Methodology: The present study utilizes an in silico 'Herbal Informatics' model which deploys dynamic search protocols, priority indexing and systemic categorization for rationale based selection of nutraceuticals targeting the critical virulence factors of Multi-Drug Resistant Salmonella enterica serovar typhimurium. Furthermore, in silico biochemical activity prediction was conducted using 'Mol inspiration' chemiinformatics tool, so as to propose the drug likeliness of selected natural plant products.

Results: Out of the 05 selected bioactivity parameters of Salmonella enterica, lipo-polysaccharide inhibition exhibited maximum relevance as physiological target i.e., $65 \%$, followed by other parameters like enterochelin inhibition, Type III secretory system inhibition, Superoxide dismutase inhibition and Symptomatic relief provision. The binary matrix analysis of database of 50 plants identified using classical bioprospection filtered 28 herbals which exhibited probable potential to mitigate 03 or more virulence factors. The weightage matrix analysis further scrutinized the selection upto 10 herbals having a score more than median weightage matrix score i.e., 14.98. The optimization of data on a scale of 0-1 using fuzzy score matrix analysis led to the final selection of 10 herbals i.e., Abrus precatorius, Azadirachta indica, Camellia sinensis, Holarrhena antidysenterica, Andrographis paniculata, Adhatoda vasica, Euphorbia hirta, Ocimum sanctum, Terminalia arjuna and Terminalia belerica. In silico Bioactivity prediction analysis of predominant phytoconstituents of selected herbals revealed Holarrhena antidysenterica (Conessine), Andrographis paniculata (Andrographolide), Euphorbia hirta (Amyrin) and Terminalia arjuna (Arjunolic acid) exhibiting drug likeliness with their targeted action as a GPCR Ligand, Nuclear Receptor Ligand or Protease inhibitor.
\end{abstract}

Keywords: Herbal Informatics; Biochemical activity; Chemiinformatics; Nosocomial; Salmonella enterica serovar typhimurium; Antibiotic resistance

\section{Introduction}

Natural Plant Products (NPPs) with medicinal potential, derived from either traditional or modern medicine, serves as a source of approx. 5000 compounds per species to fight against multifarious diseases. Such tremendous source of secondary plant metabolites employs to defend themselves against bacteria, fungi or viruses and can be used in almost the same way in medicine to treat microbial or viral infections [1]. The effective management of re-emerging pathogens with enhanced scope of attack and attributable resistance require intensive efforts in this direction. NPPs have become an ideal alternative to be explored for their therapeutic utilities by the virtue of their holistic broad spectrum activity; limited or no side effects; better acceptability and ease of local availability [2]. However, the Indian subcontinent has only $2 \%$ global market share of herbal medicine despite having 7000 Ayurvedic, 700 Unani, 600 Siddha medicines and 30 modern medicines. This clearly indicates the lack of directed research in instigating the search for newer antimicrobials based on either reverse pharmacology or classical quest of lead moiety. The expected market for NPPs is $\$ 5$ Trillion by 2050; if focused research is triggered so as to yield therapeutic leads [3]. Such focused research demands an in depth knowledge of pathophysiology of disease causing microorganisms and rationale based selection of holistic agents having bio-protective potential.

Re-emerging strains of commensal microorganisms e.g., Salmonella enterica are scourging the human population due to greater penetrance in the ecosystem and limited / no treatment options. The outbreaks of Multi-Drug Resistant (MDR) strains of Salmonella enterica serovar typhimurium in the Indian subcontinent, Southeast Asia, and Africa showed approximately 16 million cases and 60,0000 deaths each year [4]. These infections which were earlier contained within hospitals, have now higher penetration even at community level [5]. Salmonella is also associated with reactive arthritis having global population expressivity in a range of 6 to $30 \%$, out of which $0.2 \%-7.3 \%$ cases were of joint inflammation alone. Incubation period of reactive arthritis is ambiguous spanning a time period of 2-4 weeks after the onset of enteric infection [6]. Reactive arthritis is also reported to be associated with other food borne illness like Campylobacter and Shigella with reported infection rate of 1 and $4 \%$ in adults respectively [7]. Such findings implied that such infections are burdening the economic

*Corresponding author: Rajesh Arora, Scientist, Office of DG (LS), Defence Research and Development Organisation, DRDO Bhawan, Delhi, India, Tel: 01123017342; Fax: 011-23014259; E-mail: rajesharoradr@gmail.com

Received: September 30, 2015; Accepted: October 13, 2015; Published October 16, 2015

Citation: Tanwar A, Thakur P, Chawla R, Jaiswal S, Chakotiya AS, et al. (2015) Targeting Antibiotic Resistant Salmonella enterica: Bio-matrix Based Selection and Bioactivity Prediction of Potential Nutraceuticals. Biochem Anal Biochem 4: 217. doi:10.4172/2161-1009.1000217

Copyright: ( $) 2015$ Tanwar A, et al. This is an open-access article distributed under the terms of the Creative Commons Attribution License, which permits unrestricted use, distribution, and reproduction in any medium, provided the original author and source are credited. 
setup of healthcare management attributed towards a longer duration of treatment with expensive antibiotics. Resistance in multidrug resistant pathogens is accredited to chromosomal mutations and selective pressure of several synthetic drugs used for the treatment [8]. Also, the resistance profile of Salmonella enterica serovar typhimurium indicates a shift from multidrug resistance to extensive drug resistance with resistance against tetracycline, streptomycin, sulfamethoxazole, ampicillin and ceftriaxone [9]. Emergence of strains resistant to ciprofloxacin further broadened its risk spectra. The mitigation of such risk at early stages is necessary so as to cease the consequent cascade of its use with biothreat intent. New therapeutic modalities with multitargeted mode of action are needed to be investigated. The holistic mode of physiological action of natural plant products with reduced or nil adverse drug reactions might serve as the probable candidate(s) to be explored [10]

The present study utilized in silico bioprospection herbal informatics tool to filter the potential herbals targeted against virulence factors of MDR Salmonella enterica serovar typhimurium. It included web based classical data search, matrix linked data mining followed by fuzzy logic based optimization/ validation to identify potent herbals with probable therapeutic efficacy [11]. Furthermore, the selected herbals were screened for their biochemical activity at in silico level using 'Mol inspiration', a chemiinformatics tool [12]. This study hence also illustrated the active leads for ascertaining desired biological activity, which further warrants antimicrobial efficacy testing at both in vitro and in vivo levels.

\section{Methodology}

\section{Classical literature surge model}

Antibiotic resistant Salmonella enterica serovar typhimurium was selected as the target pathogen. A classical literature surge model was utilized and ascertained Enterochelin/enterobactin (ENT) Inhibition, Type III secretory system (TSSIII) Inhibition, Lipopolysaccharides (LPS) Inhibition, Superoxide dismutase (SOD) Inhibition and Symptomatic relief provision as virulence factors [13]. Similarly, the database set of herbals was selected with attributable factors as a) Ethnopharmacological importance of plant; b) Relevance of Herb in traditional medicine; c) Availability factor or cultural acceptability in localized regions; d) Any vedic literature supporting its use; e) Investigations/ prior experience on potential of the herb; f) Indirect indications, if any.

\section{Relevance factor linked binary matrix analysis}

PubMed was used as the assigned search engine for keyword hits analysis. A combination keyword converging 'anti-microbial activity and virulence factor' was entered in the query search box of PubMed. Observation based analysis of first 20 hits was done for linking the relevance of each virulence factor with its direct significance in triggering the disease [14]. Percentage relevance of each virulence factor was evaluated using the following formula;

$$
\%(\text { Relevance }) \text { avg }=\frac{(\text { No. of relevant hits based onobservational analysis })}{\text { Total No.of Hits screened }} X 100
$$

The scrutinization of primary database set of NPPs was done with respect to presence or absence of a given bioactivity parameter in the NPP, as reported in either traditional databases or PubMed search engine.

\section{Relevance factor linked weightage matrix analysis}

On the basis of average percentage relevance, each bioactivity parameter was assigned with a weightage score. Bioactivity parameter having maximum percentage relevance was assigned with a score of 05 and other succeeding parameters were provided with relative weightage scores based on unitary method evaluation. Evaluation of overall weightage of plants having a binary score $>3$ was done by multiplying their binary score with weightage score of existing bioactivity parameter. This step definitely out-ruled the possibility of any 'uncertainty factor', thereby reducing investigator's biasness. Plants with scores $\geq 3$ from previous step were only utilized to identify potent leads [15]

\section{Fuzzy set membership analysis and optimization}

The following mathematical relationship was used to ascertain relative relevance within an identified set of herbals;

$$
\mu S=\frac{(S-\operatorname{Min} S)}{(\operatorname{Max} S-\operatorname{Min} S)}
$$

Where, $\mu \mathrm{S}$ represents the desirability values of selected NPPs of the fuzzy set $\mathrm{S}$. $\operatorname{Min}(\mathrm{S})$ and $\operatorname{Max}(\mathrm{S})$ are minimum and maximum scores, respectively, in the fuzzy set $\mathrm{S}$ [16]. The estimated $\mu S$ were converted into a leveled score by using a scaled magnitude as optimization of identified potential set of NPPs.

\section{In silico biochemical and molecular activity prediction using 'Mol Inspiration'}

\section{Preparation of ligand dataset}

The 2D structures and the SMILES (Simplified Molecular-Input Line-Entry System) notation of predominant phyto-constituents of scrutinized herbals ( Fuzzy Set Score of 1 ) were obtained from Pubchem [17].

\section{Biological activity prediction}

Canonical SMILES of each of the selected biologically active phytoconstituent was considered as the test set. Based on the analysis of large training set consisting of tens of thousands of the known biologically active compounds, computer program PASS analyzed the presence or absence of specific substructures in the test set in relation to the training set. Each substructure fragment contributed towards a bioactivity score for each listed activity i.e., GPCR Ligand, Ion Channel modulator, Kinase inhibitor, Nuclear receptor ligand and Protease inhibitor. This provided a total molecule activity score (a number, typically between -3 and 3). Molecules with the highest activity score had the highest probability to be active [12].

\section{Results}

\section{Classical literature surge model}

Classical Bioprospection was done utilizing Vedic literature, ethnopharmacological databases, PubMed and direct/indirect scientific evidences as the foundation for rationale based selection of herbals targeting MDR S. enterica. Also, pathophysiology of MDR S. enterica was reviewed so as to select the crucial 05 virulence factors as drug targets (Bioactivity parameters). These bioactivity parameters were selected on the basis of certain important characteristics including a) category of microorganism as lethal, sub-lethal, incapacitating agent; b) unavailability of a treatment regime/vaccine; c) re-emerging virulent forms from past; d) intended use as a bioweapon. Similarly, classical bioprospection of 50 herbals was done on the basis of their direct significance in a) symptomatic relief provision; b) cell wall synthesis inhibition; c) bacterial enzyme inhibition; and/or d) antibiotic resistance modification. 
Citation: Tanwar A, Thakur P, Chawla R, Jaiswal S, Chakotiya AS, et al. (2015) Targeting Antibiotic Resistant Salmonella enterica: Bio-matrix Based Selection and Bioactivity Prediction of Potential Nutraceuticals. Biochem Anal Biochem 4: 217. doi:10.4172/2161-1009.1000217

\section{Relevance factor linked binary matrix analysis}

On the basis of keyword hits scoring matrix, average percentage relevance was ascertained for each virulence factor of Salmonella enterica subvar typhimurium. Rationale for selection of each of these parameters is elucidated in Table 1. Highest percentage relevance was obtained for Lipopolysaccharide inhibition $(\sim 65 \%)$, followed by other parameters like Symptomatic relief provision, Type III secretory system (TSSIII) inhibition, Superoxide dismutase (SOD) inhibition and Enterochelin/ enterobactin inhibition. Consequently weightage was given to selected parameters in the range of $0.05-5$, based on statistical unitary approach, with highest weightage i.e. 5, given to Lipopolysaccharide inhibition, followed by other parameters in decreasing order, as explicated in Table 2. The binary matrix analysis of presence / absence of these virulence factors in 50 herbals revealed that 28 herbal plants showed score $\geq 3$ i.e., the median cut off value (Table 3 ).

\section{Weightage matrix based analysis and optimization}

The weightage score matrix analysis of 28 scrutinized plants revealed that 10 plants showed highest weightage score $\geq 14.98$, thereby indicating their probable therapeutic utility against one or more of the targeted virulence factors. The final selection included Abrus precatorius, Azadirachta indica, Camellia sinensis, Holarrhena antidysenterica, Andrographis paniculata, Adhatoda vasica, Euphorbia hirta, Ocimum sanctum, Terminalia arjuna and Terminalia belerica with an optimized fuzzy score of 1.0 (Table 4).

\section{In silico biochemical activity prediction using 'mol inspira- tion'}

The bioactivity scores of the 10 scrutinized NPPs revealed that conessine (Holarrhena antidysenterica) was found to be highly bioactive $(>0.5)$ towards GPCR ligands, whereas andrographolide (Andrographis paniculata), amyrin (Euphorbia hirta) and arjunolic acid (Terminalia arjuna) were found to be highly bioactive $(>0.5)$ towards both Nuclear Receptor ligands and protease. None of the phyto-ligands were found to be exhibiting ion channel modulation and kinase inhibition. Abrin (Abrus precatorius) and Epicatechin gallate (Camellia sinensis) exhibited moderate bioactivity $(0.1 \leq$ Bioactivity Score $\leq 0.45)$ with respect to GPCR Ligand, Nuclear Receptor Ligand and Protease. Azadirachtin (Azadirachta indica), Vasicinolone

\begin{tabular}{|c|c|c|}
\hline S.No. & Bioactivity parameter & Rationale for selection (Based on Classical Approach) \\
\hline 1. & $\begin{array}{l}\text { Enterochelin/enterobactin(ENT) } \\
\text { Inhibition [23] }\end{array}$ & $\begin{array}{l}\text { - Enterochelin is a cyclic polyester consisting of three residues of } 2,3 \text {-dihydroxy-N-benzoylserine. } \\
\text { Enterobactin, a physiologically active iron sequestering agent, is excreted into the medium by } S \text {. } \\
\text { typhimurium under low iron conditions and is indispensable for growth. } \\
\text { - Enterochelin regulates the capacity of } S \text {. typhimurium to grow both in vivo and in vitro. } \\
\text { - Crude extracts of several plants like Allium sativum, Acacia catechu, } A \text {. nilotica etc have been found to be } \\
\text { bacteriostatic against multidrug resistant (MDR) strains of } S \text {. typhimurium. }\end{array}$ \\
\hline 2. & $\begin{array}{l}\text { Type III secretory system (TSSIII) } \\
\text { Inhibition } \\
\text { (Injectisome or Injectosome) [24] }\end{array}$ & $\begin{array}{l}\text { - Contains proteins that form a channel in the inner and outer bacterial membranes, as well as an } \\
\text { extracellular needle that is used for transporting and injecting effector proteins into a host cell. } \\
\text { S. typhimurium stimulates innate responses by delivering through this system, the bacterial effector } \\
\text { proteins SopE, SopE2, and SopB \& induces intestinal inflammation as well as other intestinal inflammatory } \\
\text { pathologies. } \\
\text { - Phenolic compounds from medicinal herbs and dietary plants include phenolic acids, flavonoids, tannins, } \\
\text { coumarins, lignans such as Borago officinalis, Embelis ribes, Holarrhena antidysentrica, Thymus vulgaris etc. } \\
\text { that exhibited significant effects on type III secretory system. }\end{array}$ \\
\hline 3. & $\begin{array}{l}\text { Lipopolysaccharide Inhibition } \\
\text { (LPS) [25] }\end{array}$ & $\begin{array}{l}\text { - LPS, key component of the cell wall and is required for the cellular integrity. } \\
\text { - Toxicity is due to an outer membrane consisting largely of lipo-polysaccharides (LPS) which protect the } \\
\text { bacteria from the environment. It is made up of an O-antigen (responsible for the host immune response), } \\
\text { and lipid A (made up of two phosphorylated glucosamine which are attached to fatty acids. Phosphorylation } \\
\text { of this leads to the toxicity, which connects it to the outer membrane. } \\
\text { Several plant extracts have exhibited synergistic activity like Zingiber officinalis, Semecarpus anacardium, } \\
\text { and Terminalia belerica against gram negative microorganisms and inhibits inflammation. }\end{array}$ \\
\hline 4. & $\begin{array}{l}\text { Superoxide dismutase } \\
\text { (SOD) Inhibition [26] }\end{array}$ & $\begin{array}{l}\text { - Enzymes that catalyze the dismutation of superoxide }\left(\mathrm{O}_{2}^{-}\right) \text {into oxygen and hydrogen peroxide. Human } \\
\text { WBC's generate superoxide and other reactive oxygen species to kill bacteria. } \\
\text { During infection, some bacteria therefore produce superoxide dismutase (Specially } \operatorname{sodA} \text { and } \operatorname{sodB}) \text { to } \\
\text { protect themselves from being killed, which might be helpful for the survival of strain by acting against } \\
\text { the phagocytic mechanism of the host. } \\
\text { Plants extracts that have shown synergistic effects include Terminalia belerica + Zingiber officinalis + } \\
\text { Embelia ribes to inhibit the SOD antioxidant activity of S. typhimurium. }\end{array}$ \\
\hline 5. & Symptomatic relief [27] & $\begin{array}{l}\text { - S. typhimurium is known to cause bacteremia, with or without gastrointestinal involvement; typhoid or } \\
\text { enteric fever; localized infections (e.g, bones, joints, and meninges) } \\
\text { - One of the possible disease management strategies is to alleviate the symptoms by using herbals like Vitis } \\
\text { vinifera, Piper longum, Allium sativum etc. } \\
\text { - Active ingredient such as alkaloids, flavones, glycosides, etc of medicinal herbals have been known to } \\
\text { show such symptomatic relief providing effects. }\end{array}$ \\
\hline
\end{tabular}

Table 1: Rationale for selection of the bioactivity parameters for Bioprospection study.

\begin{tabular}{|c|c|c|c|c|c|}
\hline S.No. & Bioactivity parameter & $\begin{array}{l}\text { Total No. of hits } \\
\text { screened }\end{array}$ & $\begin{array}{l}\text { Hits relevance } \\
\qquad(\mathrm{N}=20)\end{array}$ & $\begin{array}{l}\text { Percentage }(\%) \\
\text { relevance }\end{array}$ & $\begin{array}{l}\text { Relative weightage } \\
\text { assigned }\end{array}$ \\
\hline 1. & $\begin{array}{l}\text { Lipopolysaccharides inhibition } \\
\text { (LPS) }\end{array}$ & 190 & 13 & $65 \%$ & 5 \\
\hline 2. & Symptomatic relief & 33 & 9 & $45 \%$ & 3.46 \\
\hline 3. & Type III secretory system(TSSIII) inhibition & 43 & 7 & $35 \%$ & 2.69 \\
\hline 4. & $\begin{array}{l}\text { Superoxide dismutase } \\
\text { (SOD) inhibition }\end{array}$ & 50 & 6 & $30 \%$ & 2.30 \\
\hline 5. & Enterochelin/enterobactin inhibition & 32 & 4 & $20 \%$ & 1.53 \\
\hline
\end{tabular}

Table 2: Classical literature surge of virulence factors, showcasing relative percentage relevance. 
Citation: Tanwar A, Thakur P, Chawla R, Jaiswal S, Chakotiya AS, et al. (2015) Targeting Antibiotic Resistant Salmonella enterica: Bio-matrix Based Selection and Bioactivity Prediction of Potential Nutraceuticals. Biochem Anal Biochem 4: 217. doi:10.4172/2161-1009.1000217

Page 4 of 9

\begin{tabular}{|c|c|c|c|c|c|c|c|}
\hline S.No. & Plants & $\begin{array}{l}\text { Plants } \\
\text { code }\end{array}$ & $\begin{array}{l}\text { Ethnopharmacological } \\
\text { Importance }\end{array}$ & Traditional medicine & Availability & Indirect indication & $\begin{array}{l}\text { Active ingredients and chemical } \\
\text { constituents }\end{array}$ \\
\hline 1 & $\begin{array}{l}\text { Abrus precatorius } \\
\text { (Rati) [28] }\end{array}$ & Ap & $\begin{array}{l}\text { Abortifacient, anodyne, } \\
\text { aphrodisiac, antimicrobial, } \\
\text { diuretic, emetic, expectorant, } \\
\text { febrifuge, sedative }\end{array}$ & $\begin{array}{l}\text { Treatment of } \\
\text { nervous systems, } \\
\text { diarrhea, dysentery } \\
\text { and possesses } \\
\text { antihelminthic } \\
\text { properties }\end{array}$ & $\begin{array}{l}\text { Ubiquitous in } \\
\text { India,Belize, } \\
\text { Caribbean } \\
\text { Islands, Hawaii, } \\
\text { Polynesia and } \\
\text { parts of the } \\
\text { United States }\end{array}$ & $\begin{array}{l}\text { Chloroform- } \\
\text { methanol extract of } \\
\text { Abrus precatorius } \\
\text { seeds shows anti- } \\
\text { diabetic effect. It } \\
\text { is very helpful in } \\
\text { lowering the level of } \\
\text { blood sugar }\end{array}$ & $\begin{array}{l}\text { Alkaloids } 1 \% \text {, abrin, abraline, } \\
\text { choline, trigonelline, gallic acids, } \\
\text { precatorine, abricin, abridin, } \\
\text { abrectorin and several amino acids }\end{array}$ \\
\hline 2 & $\begin{array}{l}\text { Aegle marmelos } \\
(B e l) \\
{[29]}\end{array}$ & $A m$ & $\begin{array}{l}\text { Improves appetite and digestion, } \\
\text { cure for dysentery and } \\
\text { dyspepsia, fever and malaria } \\
\text { Aromatic, astringent, cooling, } \\
\text { febrifuge nature and also acts as } \\
\text { tonic for heart and brain }\end{array}$ & $\begin{array}{l}\text { Used for constipation, } \\
\text { dysentery and diarrhea }\end{array}$ & South-east Asia . & $\begin{array}{l}\text { Shows } \\
\text { hypoglycaemic } \\
\text { activities. It also } \\
\text { shows significant } \\
\text { elevation in } \\
\text { glutathione and } \\
\text { Vitamin C }\end{array}$ & $\begin{array}{l}\text { Tannins } 5 \% \text {, Mucilage } 10 \% \text { \& } \\
\text { Mucilage } 15 \% \text {, } \\
\text { Main chemical components are } \\
\text { marmelosin, alloimperatorin, } \\
\text { marmelide, tannic acid, marmin, } \\
\text { umbelliferone, isoimperatorin, } \\
\text { isopimpinellin, skimmin, marmesin, } \\
\text { marmesinin, fatty acids, and beta- } \\
\text { sitosterol }\end{array}$ \\
\hline 3 & $\begin{array}{l}\text { Acorus calamus } \\
\text { (vacha) [30] }\end{array}$ & $A c$ & $\begin{array}{l}\text { Nauseant, stomachic, } \\
\text { anthelmentic, stimulants, emetic, } \\
\text { expectorant, carminative, } \\
\text { antispasmodic and nervine } \\
\text { sedative. }\end{array}$ & $\begin{array}{l}\text { Useful in flatulence, } \\
\text { colic, dyspepsia, } \\
\text { dysentery, } \\
\text { stomach problems } \\
\text { cough, bronchitis, } \\
\text { inflammations }\end{array}$ & $\begin{array}{l}\text { Ubiqutous in } \\
\text { india and other } \\
\text { country. }\end{array}$ & $\begin{array}{l}\text { Methanolic extract of } \\
\text { rhizomes possess } \\
\text { antimicrobial activity } \\
\text { against bacteria, } \\
\text { fungi and yeast }\end{array}$ & - \\
\hline 4 & $\begin{array}{l}\text { Andrographis } \\
\text { paniculata } \\
\text { (Kalmegh) [31] }\end{array}$ & $A d p$ & $\begin{array}{l}\text { Useful in diarrhoea, dysentery, } \\
\text { enteritis, fever, cough, sore } \\
\text { throat, bronchitis, hypertension, } \\
\text { menstrual and post-partum } \\
\text { hematometra }\end{array}$ & $\begin{array}{l}\text { Anti-biotic, anti-viral, } \\
\text { anti-parasitic and } \\
\text { immune system } \\
\text { stimulant used in viral } \\
\text { hepatitis, children's } \\
\text { bowel complaints, } \\
\text { gastric acidity, liver } \\
\text { congestion and } \\
\text { flatulence }\end{array}$ & $\begin{array}{l}\text { Cultivated } \\
\text { throughout India, } \\
\text { Southern and } \\
\text { South-eastern } \\
\text { Asia }\end{array}$ & $\begin{array}{l}\text { Extract possesses } \\
\text { antioxidant and } \\
\text { anti-inflammatory } \\
\text { properties. }\end{array}$ & $\begin{array}{l}\text { Andrographolide } 5 \%-10 \% \text {. Main } \\
\text { chemical components are } \\
\text { diterpenoid lactones, paniculides, } \\
\text { farnesols, flavonoids, } \\
\text { andrographolide, andrographinin, } \\
\text { neoandrographolide and } \\
\text { isoandrographolide }\end{array}$ \\
\hline 5 & $\begin{array}{l}\text { Azadirachta indica } \\
\text { (Neem) [32] }\end{array}$ & Adi & $\begin{array}{l}\text { Anti-septic, anti-inflammatory, } \\
\text { anti-bacterial, anti-hyperglycemic } \\
\text { and insecticidal in nature. } \\
\text { Useful in vomiting, dyspepsia, } \\
\text { intestinal worms and } \\
\text { hepatopathy }\end{array}$ & $\begin{array}{l}\text { Act as vermifuge, } \\
\text { insecticide, astringent, } \\
\text { tonic and antiseptic }\end{array}$ & $\begin{array}{l}\text { Evergreen } \\
\text { and grows } \\
\text { throughout India }\end{array}$ & $\begin{array}{l}\text { Head Lice } \\
\text { treatment and } \\
\text { Measles symptoms } \\
\text { alleviation }\end{array}$ & $\begin{array}{l}\text { Bitters } 2 \% \text {, Bitters } 3 \% \text { \& Bitters } \\
5 \% \text {, azadirachtin, azadirachtol, } \\
\text { azadirachnol, desacetynimbinene, } \\
\text { nimbandiol, nimbolide, quercetin, } \\
\text { beta-sitosterol, n-hexacosanol, } \\
\text { nimbiol and nimocin }\end{array}$ \\
\hline 6 & $\begin{array}{l}\text { Adhatoda vasica } \\
\text { (Adosa) } \\
{[33]}\end{array}$ & $A v$ & $\begin{array}{l}\text { Sedative, expectorant, } \\
\text { antispasmodic and anti- } \\
\text { helminthic. It is a bronchial } \\
\text { antiseptic, bronchodilator and } \\
\text { expectorant }\end{array}$ & $\begin{array}{l}\text { Treating cold, cough, } \\
\text { whooping cough, } \\
\text { chronic bronchitis and } \\
\text { asthma. } \\
\text { Used in diarrhea and } \\
\text { dysentery }\end{array}$ & $\begin{array}{l}\text { Grows in plains } \\
\& \text { in lower } \\
\text { Himalayan } \\
\text { ranges upto } \\
1000 \text { m above } \\
\text { sea level. }\end{array}$ & $\begin{array}{l}\text { Potent antitussive } \\
\text { effect }\end{array}$ & $\begin{array}{l}\text { Vasicine } 1.0 \% \text {, Alkaloids } 1 \% \text {, } \\
2 ' \text {-hydroxy }-4 \text {-glucosyloxychalcone, } \\
\text { vasicol, adhatodine, vasicinone, } \\
\text { vasicinol, arachidic, cerotic, } \\
\text { behenic, lignoceric, linoleic and } \\
\text { oleic acids }\end{array}$ \\
\hline 7 & $\begin{array}{l}\text { Berberis aristata } \\
\text { (Daruhaldi) } \\
{[34]}\end{array}$ & $B a$ & $\begin{array}{l}\text { Anti-bacterial, anti-inflammatory, } \\
\text { astringent, alternative, } \\
\text { antipyretic, antiperiodic, anti- } \\
\text { septic, anti-cancer }\end{array}$ & $\begin{array}{l}\text { Useful in eye } \\
\text { diseases, hemorrhoids, } \\
\text { amenorrhea, } \\
\text { leucorrhoea, piles, } \\
\text { sores, peptic ulcers, } \\
\text { dysentery, heartburn, } \\
\text { indigestion, hepatitis, } \\
\text { intermittent fever, and } \\
\text { chronic ophthalmia }\end{array}$ & $\begin{array}{l}\text { Himalayan } \\
\text { region, } \\
\text { distributed } \\
\text { from kashmir to } \\
\text { uttarakhand }\end{array}$ & $\begin{array}{l}\text { Root bark is } \\
\text { anticoagulant } \\
\text { and hypotensive } \\
\text { in nature. It is } \\
\text { also used to treat } \\
\text { infections, eczema, } \\
\text { psoriasis, and } \\
\text { vaginitis }\end{array}$ & $\begin{array}{l}\text { Kerachine, Berberine, oxycanthine } \\
\text { etc }\end{array}$ \\
\hline 8 & $\begin{array}{l}\text { Camellia sinensis } \\
\text { (Green tea) } \\
{[35]}\end{array}$ & Cs & $\begin{array}{l}\text { Antibacterial, antiseptic, } \\
\text { antioxidant and detoxifying } \\
\text { properties. Helpful in curing } \\
\text { arthritis and asthma. }\end{array}$ & $\begin{array}{l}\text { It enhances the } \\
\text { liver functioning } \\
\text { and immune } \\
\text { system. Helpful in } \\
\text { mental disorders, } \\
\text { skin disorders and } \\
\text { cardiovascular } \\
\text { diseases }\end{array}$ & $\begin{array}{l}\text { Green tea } \\
\text { originated } \\
\text { in China More } \\
\text { widespread in } \\
\text { the West }\end{array}$ & $\begin{array}{l}\text { Leaves increase } \\
\text { the body resistance } \\
\text { against biological, } \\
\text { physical and } \\
\text { chemical stressors. } \\
\text { It is known as a } \\
\text { good adaptogen }\end{array}$ & $\begin{array}{l}\text { Polyphenols } 50 \%, 60 \%, 70 \% \text {, } \\
\text { caffeine, catechins, } \\
\text { epigallocatechin, epigallocatechin } \\
\text { gallate (EGCG), malic acid, oxalic } \\
\text { acid, theophylline, theobromine, } \\
\text { xanthine, inositol, kaempferol and } \\
\text { quercetin }\end{array}$ \\
\hline 8 & $\begin{array}{l}\text { Chamomile spp. } \\
\text { (Babun phool) } \\
{[36]}\end{array}$ & Cms & $\begin{array}{l}\text { Sedative, anodyne, antibacterial, } \\
\text { stomachic. }\end{array}$ & $\begin{array}{l}\text { Used as antimicrobial, } \\
\text { antihistamine, anti- } \\
\text { anxiety, diuretic }\end{array}$ & $\begin{array}{l}\text { Southern } \\
\text { Europe, Asia, } \\
\text { North America }\end{array}$ & $\begin{array}{l}\text { Chamomile is } \\
\text { frequently added } \\
\text { to skin cosmetics } \\
\text { to serve as } \\
\text { an emollient }\end{array}$ & Tannins, flavonoids resins etc \\
\hline 9. & $\begin{array}{l}\text { Euphorbia hirta } \\
\text { (Dudhi) [37] }\end{array}$ & Eh & $\begin{array}{l}\text { Bronchitic, asthma and laryngeal } \\
\text { spasm } \\
\text { anodyne, antipruritic, } \\
\text { carminative, depurative, diuretic, } \\
\text { febrifuge, galactogogue }\end{array}$ & $\begin{array}{l}\text { Used to treat intestinal } \\
\text { amoebic dysentery, } \\
\text { enteritis and skin } \\
\text { conditions }\end{array}$ & $\begin{array}{l}\text { Waste places } \\
\text { and cultivated } \\
\text { fields in lowland } \\
\text { Japan, Nepal }\end{array}$ & $\begin{array}{l}\text { Shown to kill various } \\
\text { types of pathogenic } \\
\text { bacteria, Helicobacter } \\
\text { pylori and Plasmodium }\end{array}$ & - \\
\hline
\end{tabular}


Citation: Tanwar A, Thakur P, Chawla R, Jaiswal S, Chakotiya AS, et al. (2015) Targeting Antibiotic Resistant Salmonella enterica: Bio-matrix Based Selection and Bioactivity Prediction of Potential Nutraceuticals. Biochem Anal Biochem 4: 217. doi:10.4172/2161-1009.1000217

Page 5 of 9

\begin{tabular}{|c|c|c|c|c|c|c|c|}
\hline 10 & $\begin{array}{l}\text { Ficus religiosa } \\
\text { (Peepal) } \\
{[38]}\end{array}$ & $F r$ & $\begin{array}{l}\text { Useful in inflammations } \\
\text { and glandular swellings of } \\
\text { neck. Used as astringent in } \\
\text { leucorrhoea }\end{array}$ & $\begin{array}{l}\text { Root, bark is } \\
\text { aphrodisiac and also } \\
\text { good for lumbago }\end{array}$ & $\begin{array}{l}\text { Cultivated } \\
\text { in south- } \\
\text { west China and } \\
\text { Indochina }\end{array}$ & - & - \\
\hline 11 & $\begin{array}{l}\text { Hemidesmus } \\
\text { indicus } \\
\text { (Anantmoola) } \\
{[39]}\end{array}$ & $\mathrm{Hi}$ & $\begin{array}{l}\text { Astringent, anti-inflammatory } \\
\text { properties }\end{array}$ & $\begin{array}{l}\text { Useful in treatment } \\
\text { of skin diseases, } \\
\text { wounds, psoriasis and } \\
\text { syphilis, inflammations, } \\
\text { hepatopathy and } \\
\text { neuropathy. }\end{array}$ & $\begin{array}{l}\text { Root extract } \\
\text { shows } \\
\text { potent anti- } \\
\text { enterobacterial } \\
\text { activity (S. } \\
\text { flexneri) }\end{array}$ & - & $\begin{array}{l}\text { Saponins } 5 \%, 7.5 \% \text { \& } 10 \% \text {, } \\
\text { sarsaponin, smilacin, p-methoxy } \\
\text { salicylic aldehyde, beta-sitosterol, } \\
\text { sarsapogenin, smilgenin, sitosterol, } \\
\text { stigmasterol fatty acids and tannins }\end{array}$ \\
\hline 12. & $\begin{array}{l}\text { Holarrhena } \\
\text { antidysentrica } \\
\text { (Indrajava) } \\
{[40]}\end{array}$ & $\mathrm{Ha}$ & $\begin{array}{l}\text { Astringents, antidysentric, } \\
\text { stomachic, febrifungal and tonic } \\
\text { properties }\end{array}$ & $\begin{array}{l}\text { Useful in treatment of } \\
\text { piles, skin diseases } \\
\text { and biliousness. } \\
\text { Herb for amoebic } \\
\text { dysentery and other } \\
\text { gastric disorders }\end{array}$ & $\begin{array}{l}\text { Grows wild in } \\
\text { mountains like } \\
\text { Himalayas }\end{array}$ & $\begin{array}{l}\text { Useful in } \\
\text { Rheumatoid Arthritis } \\
\text { \& Osteoarthritis. } \\
\text { Ethanolic } \\
\text { extract showed } \\
\text { antiprotozoan } \\
\text { effect against } \\
\text { Trypanosoma evani }\end{array}$ & $\begin{array}{l}\text { Alkaloids, Conamine, kurchine, } \\
\text { halorrhinene, conkurchine etc }\end{array}$ \\
\hline 13 & $\begin{array}{l}\text { Lawsonia inermis } \\
\text { (Mehandi) } \\
{[41]}\end{array}$ & $L i$ & $\begin{array}{l}\text { Useful in cephalagia, burning } \\
\text { sensation, insomnia and fever }\end{array}$ & $\begin{array}{l}\text { Protect against many } \\
\text { surface fungi and } \\
\text { bacteria and has been } \\
\text { used for its cooling and } \\
\text { astringent properties }\end{array}$ & $\begin{array}{l}\text { Northern } \\
\text { Africa, Western } \\
\text { and Southern } \\
\text { Asia }\end{array}$ & $\begin{array}{l}\text { Ethanolic extract } \\
\text { shows significant } \\
\text { wound healing } \\
\text { property }\end{array}$ & $\begin{array}{l}\text { Tannins } 5 \% \text { \& Tannins } 10 \% \text {, } \\
\text { lawsone, esculetin, fraxetin, } \\
\text { isoplumbagin, scopoletin, } \\
\text { betulin, betulinic acid, hennadiol, } \\
\text { lupeol, lacoumarin, quinone and } \\
\text { napthaquinone }\end{array}$ \\
\hline 14 & $\begin{array}{l}\text { Nelumbo nucifera } \\
\text { (Lotus) } \\
{[42]}\end{array}$ & $N n$ & $\begin{array}{l}\text { Useful in vomiting, dysentery, } \\
\text { cholera, diarrhea, ringworm } \\
\text { affection and dyspepsia, fever, } \\
\text { intermittent fever, cough, } \\
\text { burning sensation, dysuria and } \\
\text { hyperdypsia }\end{array}$ & $\begin{array}{l}\text { The rhizomes or } \\
\text { leaves are used with } \\
\text { other herbs to treat } \\
\text { sunstroke, fever, } \\
\text { diarrhoea, dysentery, } \\
\text { dizziness and vomiting } \\
\text { of blood. The whole } \\
\text { plant is used as an } \\
\text { antidote to mushroom } \\
\text { poisoning }\end{array}$ & $\begin{array}{l}\text { Tropical Asia } \\
\text { Queensland, } \\
\text { Australia } \\
\text { India }\end{array}$ & $\begin{array}{l}\text { Hydro alcoholic } \\
\text { extract of seeds is a } \\
\text { potent antioxidant. } \\
\text { It exhibits strong } \\
\text { free radical } \\
\text { scavenging activity }\end{array}$ & $\begin{array}{l}\text { Polysaccharides } 2.5 \%-5 \% \text { \& } \\
\text { Saponin } 5 \% \text {, nelumbine, nuciferine, } \\
\text { dehydronuciferine, } ß-\text {-sitosterol, } \\
\text { asparagine, nelumboside, lotusine, } \\
\text { neferine, palmitic acid, nicotinic } \\
\text { acid, glucose and amino acids }\end{array}$ \\
\hline 15 & $\begin{array}{l}\text { Ocimum sanctum } \\
\text { (Tulsi) } \\
{[43]}\end{array}$ & Os & $\begin{array}{l}\text { Diaphoretic, anti periodic, } \\
\text { stimulating, expectorant and } \\
\text { anti-catarrhal. Used in malaria, } \\
\text { bronchitis and gastric disorders }\end{array}$ & $\begin{array}{l}\text { Used as skin ointments } \\
\text { and promoted as a } \\
\text { treatment for acne }\end{array}$ & $\begin{array}{l}\text { Eastern-World } \\
\text { India and Nepal }\end{array}$ & $\begin{array}{l}\text { Extracts have shown } \\
\text { some antibacterial } \\
\text { activity } \\
\text { against } E . \text { coli, } S . \\
\text { aureus and } P . \\
\text { aeruginosa. } \\
\text { Mitigation effects of } \\
\text { radiation exposure. }\end{array}$ & $\begin{array}{l}\text { Tannins } 2 \%, 5 \%, 10 \% \text {, Urosolic } \\
\text { Acid } 1.5 \% \text {, tannins, alkaloids and } \\
\text { volatile oil }\end{array}$ \\
\hline 16. & $\begin{array}{l}\text { Piper longum } \\
\text { (Kali mirch) } \\
{[44,45]}\end{array}$ & $P I$ & $\begin{array}{l}\text { Used to treat flatulence, gout, } \\
\text { laryngitis, and paralysis, } \\
\text { abdominal tumors and gastric } \\
\text { ulcers. }\end{array}$ & $\begin{array}{l}\text { Good for aromatic, } \\
\text { stimulant, carminative, } \\
\text { constipation, } \\
\text { gonorrhea, paralysis of } \\
\text { the tongue, diarrhea, } \\
\text { cholera \& respiratory } \\
\text { infections }\end{array}$ & - & $\begin{array}{l}\text { Extract possesses } \\
\text { significant } \\
\text { immunomodulatory, } \\
\text { anti-arthritic and } \\
\text { anti-tumor activity }\end{array}$ & $\begin{array}{l}\text { Piperine } 3 \% \text {, Rutin } 4 \%-6 \% \text {, } \\
\text { beta- caryophyllene piperyline, } \\
\text { piperoleines }\end{array}$ \\
\hline 17. & $\begin{array}{l}\text { Syzygium cumini } \\
\text { (Jamun) } \\
{[46]}\end{array}$ & Syc & $\begin{array}{l}\text { Antidiabetic agent by decreasing } \\
\text { kidney's catalase activity, } \\
\text { Antihelminthic, Astringent }\end{array}$ & $\begin{array}{l}\text { Useful in diabetes, } \\
\text { diarrhoea and } \\
\text { ringworm infection. } \\
\text { Leaves and bark are } \\
\text { used for controlling } \\
\text { blood pressure } \\
\text { and gingivitis }\end{array}$ & $\begin{array}{l}\text { Bangladesh, } \\
\text { India, Nepal, } \\
\text { Sri Lanka, } \\
\text { Indonesia }\end{array}$ & $\begin{array}{l}\text { Bark extract } \\
\text { possesses potent } \\
\text { anti-inflammatory } \\
\text { activity }\end{array}$ & $\begin{array}{l}\text { Bitter } 3 \% \text {, Bitter } 5 \% \text { \& Volatile Oil } \\
1 \% \text {, methylxanthoxylin, corilagin, } \\
\text { ellagitanins, ellagic acid and } \\
\text { gallic acid, jambosine, volatile oil, } \\
\text { jambolin, quercetin, ferulic acid, } \\
\text { veratrole, guajacol and caffeic acid }\end{array}$ \\
\hline 18. & $\begin{array}{l}\text { Terminalia arjuna } \\
\text { (Arjuna) [47] }\end{array}$ & Taj & $\begin{array}{l}\text { Astringent, sweet, acrid, cooling, } \\
\text { aphrodisiac, cardiotonic, urinary } \\
\text { astringent, expectorant, alexiteric } \\
\text { and is useful in fractures, ulcers, } \\
\text { cirrhosis of the liver, hyperhidsis, } \\
\text { otalgia and hypertension }\end{array}$ & $\begin{array}{l}\text { It improves cardiac } \\
\text { muscle function and } \\
\text { pumping action of } \\
\text { the heart. Useful } \\
\text { for asthma, bile duct } \\
\text { disorders, scorpion } \\
\text { stings, and poisonings }\end{array}$ & $\begin{array}{l}\text { West Bengal and } \\
\text { south and } \\
\text { central India }\end{array}$ & $\begin{array}{l}\text { Bark shows potent } \\
\text { anti-anginal and } \\
\text { cardio-protective } \\
\text { activity }\end{array}$ & $\begin{array}{l}\text { Arjunolic Acid } 0.5 \% \text {, Tannin } 25 \% \text {, } \\
\text { tannins, triterpenoid saponins } \\
\text { (arjunic acid, arjunolic acid, } \\
\text { arjungenin and arjunic acid), } \\
\text { flavonoids, gallic acid, ellagic acid } \\
\text { and phytosterols }\end{array}$ \\
\hline 19. & $\begin{array}{l}\text { Terminalia } \\
\text { belerica } \\
\text { (Behada) [48] }\end{array}$ & $T b$ & $\begin{array}{l}\text { It is astringent, tonic, expectorant } \\
\text { and laxative }\end{array}$ & $\begin{array}{l}\text { Useful in cough, } \\
\text { bronchitis and } \\
\text { pharyngitis. Used in } \\
\text { dyspepsia, flatulence, } \\
\text { dipsia, and vomiting }\end{array}$ & $\begin{array}{l}\text { Ubiquitous in } \\
\text { India } \\
\text { Southeast Asia, }\end{array}$ & $\begin{array}{l}\text { Extract reduces the } \\
\text { levels of lipids in } \\
\text { hypercholesterolemia } \\
\text { models. }\end{array}$ & $\begin{array}{l}\text { Tannins } 40 \% \text { \& Tannins } 60 \% \text {, } 3- \\
\text { sitosterol, gallic acid, ellagic acid, } \\
\text { ethyl gallate, galloyl glucose and } \\
\text { chebulaginic acid }\end{array}$ \\
\hline
\end{tabular}


Citation: Tanwar A, Thakur P, Chawla R, Jaiswal S, Chakotiya AS, et al. (2015) Targeting Antibiotic Resistant Salmonella enterica: Bio-matrix Based Selection and Bioactivity Prediction of Potential Nutraceuticals. Biochem Anal Biochem 4: 217. doi:10.4172/2161-1009.1000217

Page 6 of 9

\begin{tabular}{|c|c|c|c|c|c|c|c|}
\hline 20. & $\begin{array}{l}\text { Thymus vulgaris } \\
\text { (Thyme) } \\
\text { [49] }\end{array}$ & $T V$ & $\begin{array}{l}\text { Anthelmintic, strongly antiseptic, } \\
\text { antispasmodic, carminative, } \\
\text { deodorant, diaphoretic, } \\
\text { disinfectant, expectorant, } \\
\text { sedative and tonic }\end{array}$ & $\begin{array}{l}\text { Treatment of dry } \\
\text { coughs, whooping } \\
\text { cough, bronchitis, } \\
\text { bronchial catarrh, } \\
\text { asthma, laryngitis, } \\
\text { indigestion, gastritis } \\
\text { and diarrhea }\end{array}$ & $\begin{array}{l}\text { Indigenous to } \\
\text { mediterranean } \\
\text { regions and } \\
\text { southern europe, } \\
\text { but prospers } \\
\text { almost anywhere } \\
\text { in temperate } \\
\text { climate }\end{array}$ & $\begin{array}{l}\text { Extract shown } \\
\text { antibacterial activity } \\
\text { against the gram } \\
\text { negative bacteria }\end{array}$ & $\begin{array}{l}\text { Main chemical components } \\
\text { are a-thujone, a-pinene, and } \\
\text { camphene }\end{array}$ \\
\hline 21 & $\begin{array}{l}\text { Vitis vinifera } \\
\text { (Angoor) } \\
{[50]}\end{array}$ & $V v$ & $\begin{array}{l}\text { Used for the treatment } \\
\text { of cancer, cholera, smallpox, nausea, } \\
\text { skin and eye infections as well as } \\
\text { kidney and liver diseases }\end{array}$ & $\begin{array}{l}\text { Leaves being used to } \\
\text { stop bleeding, pain and } \\
\text { inflammation }\end{array}$ & $\begin{array}{l}\text { Central Europe } \\
\text { south- } \\
\text { western Asia }\end{array}$ & $\begin{array}{l}\text { Used as anti- } \\
\text { inflammatory and } \\
\text { antibacterial agents }\end{array}$ & Phenolic compounds \\
\hline 22 & $\begin{array}{l}\text { Allium satium } \\
\text { (Garlic) [51] }\end{array}$ & As & $\begin{array}{l}\text { Antiseptic and antimicrobial } \\
\text { activity }\end{array}$ & $\begin{array}{l}\text { Use to prevent scurvy, } \\
\text { because of its high } \\
\text { vitamin } \mathrm{C} \text { content }\end{array}$ & $\begin{array}{l}\text { Asia, South Asia, } \\
\text { Southeast Asia, } \\
\text { Africa, Europe }\end{array}$ & $\begin{array}{l}\text { Extract yields allicin, } \\
\text { an antibiotic and } \\
\text { antifungal } \\
\text { compound }\end{array}$ & $\begin{array}{l}\text { Vitamins, proteins, minerals, sapo- } \\
\text { nins, flavonoids. }\end{array}$ \\
\hline 23 & $\begin{array}{l}\text { Zingiber officinalis } \\
\text { (Ginger) [52] }\end{array}$ & Zo & $\begin{array}{l}\text { Clinically proven as prophylactic } \\
\text { of nausea and vomiting } \\
\text { associated with motion, } \\
\text { sickness, sea sickness and } \\
\text { pregnancy } \\
\text { Known for its gastrointestinal } \\
\text { benefits and as an anti } \\
\text { inflamatory and carminative. }\end{array}$ & $\begin{array}{l}\text { Used in dyspepsia, } \\
\text { diarrhoea, colic, } \\
\text { flatulence, vomiting, } \\
\text { nausea, cough, cold, } \\
\text { asthma }\end{array}$ & $\begin{array}{l}\text { South east Asia } \\
\text { and throughout } \\
\text { India }\end{array}$ & $\begin{array}{l}\text { Rhizome is a potent } \\
\text { inhibitor of platelet } \\
\text { activation and } \\
\text { aggregation }\end{array}$ & $\begin{array}{l}\text { Gingerols } 5 \% \text {, gingeole, zingerone, } \\
\text { shogaol, zingiberene, cineol, } \\
\text { borneol, phellandrene, citral, } \\
\text { zingiberene, linalool, geraniol, } \\
\text { chavicol, vanillyl alcohol, } \\
\text { camphene and resin }\end{array}$ \\
\hline 24 & $\begin{array}{l}\text { Acacia nilotica } \\
\text { [53] }\end{array}$ & $A n$ & $\begin{array}{l}\text { Strongly astringent due to tannin } \\
\text { algicidal activity }\end{array}$ & $\begin{array}{l}\text { Useful in treatment of } \\
\text { diarrhea, dysentery, } \\
\text { fever, gallbladder, } \\
\text { hemorrhage, } \\
\text { hemorrhoids, } \\
\text { leucorrhea }\end{array}$ & $\begin{array}{l}\text { Zanzibar, } \\
\text { Pemba, and } \\
\text { India; Arabia }\end{array}$ & $\begin{array}{l}\text { Algicidal activity } \\
\text { against Chroccoccus, } \\
\text { Closteruim, } \\
\text { Coelastrum, } \\
\text { Cosmarium }\end{array}$ & $\begin{array}{l}\text { Sulphoxides pentosan, saponin, } \\
\text { tannin. Seeds contain crude protein } \\
18.6 \% \text {, ether extract } 4.4 \% \text {, fiber } \\
10.1 \% \text {, nitrogen-free extract } 61.2 \%\end{array}$ \\
\hline 25 & $\begin{array}{l}\text { Withania } \\
\text { somnifera(Ashwa } \\
\text { gandha) [54] }\end{array}$ & Ws & $\begin{array}{l}\text { Used as tonic, abortifacient, } \\
\text { astringent, deobstruent, nervine, } \\
\text { aphrodisiac and sedative }\end{array}$ & $\begin{array}{l}\text { Rheumatism, leprosy } \\
\text { and arthritis }\end{array}$ & $\begin{array}{l}\text { Dried regions } \\
\text { of India, such as } \\
\text { Punjab, Sindh, and } \\
\text { Rajasthan. It is also } \\
\text { found in Nepal }\end{array}$ & $\begin{array}{l}\text { Inhibits the growth } \\
\text { of human tumor cell } \\
\text { line. It increases } \\
\text { the levels of } \\
\text { corticosterone in the } \\
\text { adrenal glands of } \\
\text { stress }\end{array}$ & $\begin{array}{l}\text { Alkaloids } 1 \% \text {, Alkaloids } 2 \% \text {, } \\
\text { Withanolides } 1.5 \% \text { \& Withanolides } \\
2 \% \text {, glycosides, withanolides, } \\
\text { withasomnine, hentriacontane, } \\
\text { dulcitol, withaniol and withaferin }\end{array}$ \\
\hline 26 & $\begin{array}{l}\text { Acacia catechu } \\
\text { [55] }\end{array}$ & $A c$ & $\begin{array}{l}\text { Strong antioxidant, astringent, } \\
\text { anti-inflammatory, anti-bacterial } \\
\text { and anti-fungal in nature }\end{array}$ & $\begin{array}{l}\text { Useful in stomach } \\
\text { problems like diarrhea, } \\
\text { dysentery, colitis and } \\
\text { gastric cancer }\end{array}$ & $\begin{array}{l}\text { Asia, China, } \\
\text { India and } \\
\text { the Indian } \\
\text { Ocean area }\end{array}$ & $\begin{array}{l}\text { Extract has } \\
\text { significant } \\
\text { antipyretic, } \\
\text { antidiarrhoeal, } \\
\text { hypoglycaemic and } \\
\text { hepatoprotective } \\
\text { properties }\end{array}$ & $\begin{array}{l}\text { Tannins } 25 \% \text {, Catechin } 10 \% \text {, } \\
\text { catechuic acid, pyrocatechin, } \\
\text { phloroglucin, protocatechuic acid, } \\
\text { quercetin, gum and minerals }\end{array}$ \\
\hline 27 & $\begin{array}{l}\text { Semecarpus } \\
\text { anacardium } \\
\text { (Bhalltak) } \\
{[56]}\end{array}$ & Sa & $\begin{array}{l}\text { Useful in leucoderma, scaly skin, } \\
\text { allergic, dermatitis, poisonous } \\
\text { bites, leprosy, cough, asthma, } \\
\text { and dyspepsia }\end{array}$ & $\begin{array}{l}\text { Act as insecticides, } \\
\text { antiseptic, termite } \\
\text { repellents and } \\
\text { herbicide }\end{array}$ & India & - & Anacardic acid $0.4 \%$, Proteins \\
\hline 28 & $\begin{array}{l}\text { Punica granatum } \\
\text { (Anar) [57] }\end{array}$ & $P g$ & $\begin{array}{l}\text { Antioxidant, Anticarcinogenic } \\
\text { and anti-inflammatory properties }\end{array}$ & $\begin{array}{l}\text { Stomach carcinoma } \\
\text { and syphilis treatment }\end{array}$ & $\begin{array}{l}\text { Cultivated in } \\
\text { India }\end{array}$ & $\begin{array}{l}\text { Seed extract } \\
\text { possess hypo- } \\
\text { glycaemic activity }\end{array}$ & $\begin{array}{l}\text { Ellagic Acid } 20 \% \text {, Ellagic Acid } 40 \% \\
\& \text { Tannins } 25 \% \text {, punicalagin, ellagic } \\
\text { acid, luteolin etc }\end{array}$ \\
\hline
\end{tabular}

Table 3: Herbal plants showing probable utility against Salmonella enterica serovar typhimurium infections.

\begin{tabular}{|c|c|c|c|c|c|}
\hline S.No. & Herbal Plant & Herbal code & Binary score & Weightage score & Fuzzy score $(\mu S)$ \\
\hline 1 & Andrographis paniculata & $A p$ & 5 & 14.98 & 1 \\
\hline 2 & Azadirachta indica & $A i$ & 5 & 14.98 & 1 \\
\hline 3 & Adhatoda vasica & $A v$ & 5 & 14.98 & 1 \\
\hline 4 & Euphorbia hirta & $E h$ & 5 & 14.98 & 1 \\
\hline 5 & Ocimum sanctum & Os & 5 & 14.98 & 1 \\
\hline 6 & Terminalia arjuna & $\mathrm{Ta}$ & 5 & 14.98 & 1 \\
\hline 7 & Terminalia belerica & $T b$ & 5 & 14.98 & 1 \\
\hline 8 & Abrus precatorius & $A p$ & 5 & 14.98 & 1 \\
\hline 9 & Camellia sinensis & Cs & 5 & 14.98 & 1 \\
\hline 10 & Holarrhena antidysenterica & $\mathrm{Ha}$ & 5 & 14.98 & 1 \\
\hline 11 & Aegle marmelos & $A m$ & 4 & 13.45 & 0.867 \\
\hline 12 & Vitis vinifera & $V v f$ & 4 & 13.45 & 0.867 \\
\hline 13 & Acacia catechu & $A c$ & 4 & 12.68 & 0.8 \\
\hline 14 & Berberis aristata & $B a$ & 4 & 12.68 & 0.8 \\
\hline 15 & Ficus religiosa & $F r$ & 4 & 12.68 & 0.8 \\
\hline
\end{tabular}




\begin{tabular}{|c|c|c|c|c|c|}
\hline 16 & Punica granatum & $P g$ & 4 & 12.68 & 0.8 \\
\hline 17 & Nelimbo nucifera & $N n$ & 4 & 12.68 & 0.8 \\
\hline 18 & Rosmarinus officinalis & Ro & 4 & 12.68 & 0.8 \\
\hline 19 & Syzygium cumini & Syc & 4 & 12.68 & 0.8 \\
\hline 20 & Chamomile spp. & $\mathrm{Cms}$ & 4 & 12.68 & 0.8 \\
\hline 21 & Zingiber officinale & Zo & 4 & 12.68 & 0.8 \\
\hline 22 & Acacia nilotica & $A n$ & 4 & 12.68 & 0.8 \\
\hline 23 & Hemidemus indicus & $H i$ & 4 & 12.68 & 0.8 \\
\hline 24 & Withania somnifera & Ws & 4 & 12.68 & 0.8 \\
\hline 25 & Acorus calamus & $A c$ & 4 & 12.68 & 0.8 \\
\hline 26 & Semecarpus anacardium & Sa & 4 & 12.68 & 0.8 \\
\hline 27 & Lawsonia inermis & $\mathrm{Li}$ & 4 & 12.68 & 0.8 \\
\hline 28 & Thymus vulgaris & $T V$ & 4 & 9.98 & 0.567 \\
\hline
\end{tabular}

Table 4: Fuzzy Set Membership Analysis for herbal plants screened on the basis of Binary and Weightage Matrix scores.

\begin{tabular}{|c|c|c|c|c|c|c|c|c|}
\hline S.No & Herbal Plant & $\begin{array}{c}\text { Predominant } \\
\text { Phytoconstituent }\end{array}$ & $\begin{array}{l}\text { GPCR } \\
\text { Ligand }\end{array}$ & $\begin{array}{l}\text { Ion Channel } \\
\text { Modulator }\end{array}$ & $\begin{array}{l}\text { Kinase } \\
\text { Inhibitor }\end{array}$ & $\begin{array}{l}\text { Nuclear } \\
\text { Receptor } \\
\text { Ligand }\end{array}$ & $\begin{array}{l}\text { Protease } \\
\text { Inhibitor }\end{array}$ & Inference \\
\hline 1 & $\begin{array}{c}\text { Holarrhena } \\
\text { antidysenterica }\end{array}$ & Conessine & 0.63 & 0.13 & -0.32 & 0.25 & 0.51 & $\begin{array}{c}\text { GPCR Ligand and Protease } \\
\text { inhibition activity }\end{array}$ \\
\hline 2 & $\begin{array}{l}\text { Andrographis } \\
\text { paniculata }\end{array}$ & Andrographolide & 0.32 & 0.17 & -0.01 & 0.94 & 0.81 & $\begin{array}{l}\text { Nuclear Receptor Ligand and } \\
\text { Protease inhibition activity }\end{array}$ \\
\hline 3 & Euphorbia hirta & Amyrin & 0.22 & -0.05 & -0.31 & 0.67 & 0.56 & $\begin{array}{l}\text { Nuclear Receptor Ligand and } \\
\text { Protease inhibition activity }\end{array}$ \\
\hline 4 & Terminalia arjuna & Arjunolic acid & 0.20 & -0.22 & -0.38 & 0.80 & 0.63 & $\begin{array}{l}\text { Nuclear Receptor Ligand and } \\
\text { Protease inhibition activity }\end{array}$ \\
\hline 5 & Camellia sinensis & Epicatechin gallate & 0.17 & 0.02 & 0.05 & 0.34 & 0.13 & Moderate activity \\
\hline 6 & Abrus precatorius & Abrin & 0.45 & 0.44 & -0.03 & -0.36 & 0.27 & Moderate activity \\
\hline 7 & Azadirachta indica & Azadirachtin & -0.71 & -1.51 & -1.46 & -0.67 & -0.35 & No Bioactivity \\
\hline 8 & Adhatoda vasica & Vasicinolone & -0.25 & -0.25 & -0.33 & -0.51 & -0.87 & No Bioactivity \\
\hline 9 & Ocimum sanctum & Eugenol & -0.86 & -0.36 & -1.14 & -0.78 & -1.29 & No Bioactivity \\
\hline 10 & Terminalia belerica & Chebulagic acid & -3.51 & -4.13 & -4.15 & -4.00 & -3.24 & No Bioactivity \\
\hline
\end{tabular}

Table 5: In Silico bioactivity scores of selected natural plant products.

(Adhatoda vasica), Eugenol (Ocimum sanctum) and Chebulagic acid (Terminalia belerica) were found to exhibit nil bioactivity with respect to all the bioactivity predictors (Table 5 ). Final categorized NPPs on the basis of matrix based selection and in silico biochemical analysis were Holarrhena antidysenterica, Andrographis paniculata, Euphorbia hirta, Terminalia arjuna, Abrus precatorius and Camellia sinensis.

\section{Discussion}

Antibiotics, the defense resort against the sturdy pathogens of commensal as well as nosocomial origin, are now losing the battle against multi-drug resistant re-emerging pathogens e.g., Salmonella enterica serovar typhimurium (diversified food borne pathogen) [18]. Such conflict has arisen due to the irrational usage of antibiotics, thereby transforming commensals into extensively drug resistant forms. Natural selection and sudden mutations might be conferring resistance in these pathogens [8]. The present day synthetic drugs are not competent enough to overcome the irreversible effects of such resistance patterns. Limitations of chemotherapeutic moieties i.e., systemic toxicity and adverse drug reactions, hence require to be outruled by instigating the search for newer antimicrobials of plant origin having holistic potential [1].

The present study utilizes an ethnopharmacological approach amalgamated with informatics for rationale based selection of herbals. Random search model approach was devised using PubMed as the search engine. 05 bioactivity parameters were selected on the basis of their direct/ indirect role in a) symptomatic relief provision, b) antibiotic resistance modification, c) bacterial enzyme modification, d) cell wall synthesis inhibition (Table 1). This bioprospection study was found to be in consonance with a similar study of the evaluation of sleep wake cycle in healthy individuals conducted by Elizabeth S. Jenuwine et al. [19]. The importance of herbal informatics model can further be viewed from the fact that herbals have been used as the main reservoir for a variety of natural plant products having bio-protection abilities e.g., Taxol (anticancerous, Taxus baccata), Silymarin (radioprotector, Silybum mariannum), Vincristine (anticancerous, Rosmarinus officinalis), Vinblastin (anticancerous, Rosmarinus officinalis) etc [20].

Binary coefficient matrix analysis, the first screening step of herbal informatics, was used to identify herbals on the basis of all or none principle, thereby eliminating the outliers which have scored less than the median cut off value i.e., $>3$. After the first screening step, 28 plants were filtered from a pool of 50 plants, thus reducing the total time of execution. Furthermore, weightage matrix based analysis was used to scrutinize the selection process in case of 02 or more herbals with identical binary score. Final decision of selection of herbals was based on fuzzy set membership analysis, so as to provide a universally acceptable optimized score [16].

The final categorized set of plants included Abrus precatorius (Rati), Azadirachta indica (Neem), Camellia sinensis (Green tea), Holarrhena antidysenterica (Indrajava), Andrographis paniculata (Kalmegh), Adhatoda vasica (Adosa), Euphorbia hirta (Dudhi), Ocimum sanctum (Tulsi), Terminalia arjuna (Arjuna) and Terminalia belerica (Behada). All these above mentioned plants have been reported to be contain a variety of secondary metabolites like conessine (alkaloid, Holarrhena antidysenterica); andrographolide (alkaloid, Andrographis paniculata) and epicatechin gallate (flavonoid, Camellia sinensis) etc [1,20]. 
Citation: Tanwar A, Thakur P, Chawla R, Jaiswal S, Chakotiya AS, et al. (2015) Targeting Antibiotic Resistant Salmonella enterica: Bio-matrix Based Selection and Bioactivity Prediction of Potential Nutraceuticals. Biochem Anal Biochem 4: 217. doi:10.4172/2161-1009.1000217

Furthermore, the chemiinformatics based biochemical activity prediction of predominant phytoconstituents of these NPPs gave an insight of their mechanistic mode of action. Phytoconstituents which were exhibiting bioactivity with respect to either GPCR or Nuclear receptor ligand (i.e., Conessine, Andrographolide, Amyrin, Arjunolic acid) might have a direct or indirect role in regulation of immune system activity and inflammation. Both $G$ protein-coupled receptor signaling and Nuclear Receptor binding lead to mast cell degranulation which subsequently induces localized inflammation and bacterial antigen clearance [21]. Moreover secondary metabolites with bioactivity linked to protease inhibition (e.g., Conessine, Andrographolide, Amyrin, Arjunolic acid) is directly contemplating the antimicrobial activity of these phytoconstituents as protease is known to be a key enzyme of bacteria required for evasion of host immune defenses, nutrients acquisition for growth and proliferation and tissue necrosis [22]. These secondary metabolites of NPPs might therefore, be responsible for their holistic antibacterial action, which can further be tested and validated at in vitro and in vivo levels.

\section{Conclusion}

The study has provided 06 NPPs i.e., Holarrhena antidysenterica, Andrographis paniculata, Euphorbia hirta, Terminalia arjuna, Abrus precatorius and Camellia sinensis with significant therapeutic potential that need to be explored at in vitro and pre-clinical level to manage MDR Salmonella enterica serovar typhimurium and other related infections.

\section{Acknowledgements}

The authors are grateful to Director, INMAS and Vice Chancellor, Jamia Hamdard for provision of research facilities and support. The present study was conducted under the Project number INM-318 of 'Samarthya' Programme of DRDO.

\section{References}

1. Sibanda T, Okoh Al (2007) The challenges of overcoming antibiotic resistance: Plant extracts as potential sources of antimicrobial and resistance modifying agents. African J Biotechnol 6: 2886-2896.

2. Wichtl M (2004) Herbal Drugs and Phytopharmaceuticals: A Handbook for Practice on a Scientific Basis. CRC Press.

3. Bhattacharya R, Reddy KRC, Mishra AK (2014) Export strategy of Ayurvedic Products from India. Int J Ayurvedic Med 5: 125-128.

4. Mead PS, Slutsker L, Dietz V, McCaig LF, Bresee JS, et al. (1999) Food-related illness and death in the United States. Emerg Infect Dis 5: 607-625.

5. Thomas MK, Majowicz SE, Sockett PN, Fazil A, Pollari F, et al. (2006) Estimated Numbers of Community Cases of Illness Due to Salmonella, Campylobacter and Verotoxigenic Escherichia Coli: Pathogen-specific Community Rates. Can $\mathrm{J}$ Infect Dis Med Microbiol 17: 229-234.

6. Inman RD, Johnston ME, Hodge M, Falk J, Helewa A (1988) Postdysenteric reactive arthritis. A clinical and immunogenetic study following an outbreak of salmonellosis. Arthritis Rheum 31: 1377-1383.

7. Simonet ML (1999) Enterobacteria in reactive arthritis: Yersinia, Shigella, and Salmonella. Rev Rhum Engl Ed 66: 14S-18S.

8. Guilhelmelli F, Vilela N, Albuquerque P, Derengowski L da S, Silva-Pereira I, et al (2013) Antibiotic development challenges: The various mechanisms of action of antimicrobial peptides and of bacterial resistance. Front Microbiol 4: 1-12.

9. Magiorakos A-P, Srinivasan A, Carey RB, Carmeli Y, Falagas ME, et al. (2012) Multidrug-resistant, extensively drug-resistant and pandrug-resistant bacteria: an international expert proposal for interim standard definitions for acquired resistance. Clin Microbiol Infect 18: 268-281.

10. Thakur P, Chawla R, Goel R, Narula A, Shakya SK, et al. (2015) In-Silico Bioprospection Approach for Targeting Infections Caused by Multi-Drug Resistant Proteus vulgaris. Int J Pharm Int Life Sci 1: 1-7.

11. Thakur P, Chawla R, Goel R, Arora R, Sharma RK (2013) In silico modeling for Identification of promising antimicrobials of Herbal origin against highly virulent pathogenic strains of bacteria like New Delhi Metallo-beta-lactamase -1 Escherichia coli. Int J Innov Appl 4: 2028-9324.

12. Mullaicharam A, Halligudi $\mathrm{N}, \mathrm{Al}$-bahri $\mathrm{H}$ (2012) Molecular modifications of ibuprofen using Insilico modeling system. Int J Nutr Pharmacol Neurol Dis 2: 156.

13. van Asten AJ, van Dijk JE (2005) Distribution of "classic" virulence factors among Salmonella spp. FEMS Immunol Med Microbiol 44: 251-259.

14. Singh Chakotiya A, Chawla R, Tomar M, Thakur P, Goel R, et al (2014) In silico Herbal Bioprospection targeting Multi-drug resistant Pseudomonas aeruginosa. Int J Interdiscip Multidiscip Stud 2: 163-176.

15. Thakur P, Chawla R, Goel R, Narula A, Arora R, et al. (2015) Herbal Informatics Approach for targeting Nosocomial Infections caused by Klebsiella pneumoniae. Int J Interdiscip Multidiscip Stud 2: 173-185.

16. Meimandipour A, Hosseini SA, Lotfolahian $\mathrm{H}$, Hosseini SJ, Hosseini $\mathrm{SH}$, et al. (2012) Multiattribute decision-making: use of scoring methods to compare the performance of laying hen fed with different level of yeast. Ital J Anim Sci 11: 82-86.

17. Oprea TI, Tropsha A (2006) Target, chemical and bioactivity databases integration is key. Drug Discov Today Technol 3: 357-365.

18. Threlfall EJ, Ward LR, Frost JA, Willshaw GA (2000) The emergence and spread of antibiotic resistance in food-borne bacteria. Int J Food Microbiol 62: 1-5.

19. Jenuwine ES, Floyd JA (2004) Comparison of Medical Subject Headings and text-word searches in MEDLINE to retrieve studies on sleep in healthy individuals. J Med Libr Assoc 92: 349-353.

20. Harvey AL (2008) Natural products in drug discovery. Drug Discov Today 13 894-901.

21. Kuehn HS, Gilfillan AM (2007) G protein-coupled receptors and the modification of FcepsilonRI-mediated mast cell activation. Immunol Lett 113: 59-69.

22. Mushi NF, Mbwambo ZH, Innocent E, Tewtrakul S (2012) Antibacterial, anti-HIV-1 protease and cytotoxic activities of aqueous ethanolic extracts from Combretum adenogonium Steud. Ex A. Rich (Combretaceae). BMC Complement Altern Med 12: 163.

23. Yancey RJ, Breeding SA, Lankford CE (1979) Enterochelin (enterobactin): virulence factor for Salmonella typhimurium. Infect Immun 24: 174-180.

24. Kubori T, Matsushima Y, Nakamura D, Uralil J, Lara-Tejero M, et al. (1998) Supramolecular structure of the Salmonella typhimurium type III protein secretion system. Science 280: 602-605.

25. Osborn Mj (1963) Studies On The Gram-Negative Cell Wall. I. Evidence For The Role Of 2-Keto- 3-Deoxyoctonate In The Lipopolysaccharide Of Salmonella Typhimurium. Proc Natl Acad Sci U S A 50: 499-506.

26. Canvin J, Langford PR, Wilks KE, Kroll JS (1996) Identification of sodC encoding periplasmic $[\mathrm{Cu}, \mathrm{Zn}]$-superoxide dismutase in Salmonella. FEMS Microbiol Lett 136: 215-220.

27. Duke JA (1997) The Green Pharmacy: New Discoveries in Herbal Remedies for Common Diseases and Conditions from the World's Foremost Authority on Healing Herbs. Rodale

28. Nwodo OF, Alumanah EO (1991) Studies on Abrus precatorius seeds. II: Antidiarrhoeal activity. J Ethnopharmacol 31: 395-398.

29. Arul V, Miyazaki S, Dhananjayan R (2005) Studies on the anti-inflammatory, antipyretic and analgesic properties of the leaves of Aegle marmelos Corr. Ethnopharmacol 96: 159-163.

30. Motley TJ (1994) The ethnobotany of sweet flag,acorus Calamus (Araceae) Econ Bot 48: 397-412.

31. Thakur P, Chawla R, Goel R, Narula A, Arora R, et al. (2015) Antibacterial activity of aquo-alcoholic extract of camellia sinensis against isolates of Carbapenem Resistant Escherichia coli and food borne pathogens. Int J Biol Pharm Res 6: 606-616.

32. van der Nat JM, van der Sluis WG, de Silva KT, Labadie RP (1991) Ethnopharmacognostical survey of Azadirachta indica A. Juss (Meliaceae). J Ethnopharmacol 35: 1-24.

33. Claeson UP, Malmfors T, Wikman G, Bruhn JG (2000) Adhatoda vasica: a critica review of ethnopharmacological and toxicological data. J Ethnopharmacol 72: 1-20.

34. Singh M, Srivastava S, Rawat AK (2007) Antimicrobial activities of Indian Berberis species. Fitoterapia 78: 574-576. 
Citation: Tanwar A, Thakur P, Chawla R, Jaiswal S, Chakotiya AS, et al. (2015) Targeting Antibiotic Resistant Salmonella enterica: Bio-matrix Based Selection and Bioactivity Prediction of Potential Nutraceuticals. Biochem Anal Biochem 4: 217. doi:10.4172/2161-1009.1000217

Page 9 of 9

35. Thakur P, Chawla R, Chakotiya A, Nagpal R, et al. (2015) In vitro Antibacterial Activity of Camellia sinensis and Andrographis paniculata against Vancomycin Resistant Enterococci. Int J Pharm Sci Drug Res 7: 157-162.

36. Sebai H, Jabri MA, Souli A, Rtibi K, Selmi S, et al. (2014) Antidiarrheal and antioxidant activities of chamomile (Matricaria recutita L.) decoction extract in rats. J Ethnopharmacol 152: 327-332.

37. Kumar S, Malhotra R, Kumar D (2010) Euphorbia hirta: Its chemistry, traditional and medicinal uses, and pharmacological activities. Pharmacogn Rev 4: 58-61.

38. Singh D, Singh B, Goel RK (2011) Traditional uses, phytochemistry and pharmacology of Ficus religiosa: a review. J Ethnopharmacol 134: 565-583.

39. Mary NK, Achuthan CR, Babu BH, Padikkala J (2003) In vitro antioxidant and antithrombotic activity of Hemidesmus indicus (L) R.Br. J Ethnopharmacol 87: 187-191.

40. Chakraborty A, Brantner AH (1999) Antibacterial steroid alkaloids from the stem bark of Holarrhena pubescens. J Ethnopharmacol 68: 339-344.

41. Hsouna A Ben, Trigui M, Culioli G, Blache Y, Jaoua S (2011) Antioxidant constituents from Lawsonia inermis leaves: Isolation, structure elucidation and antioxidative capacity. Food Chem 125: 193-200.

42. Huang B, Ban X, He J, Tong J, Tian J, et al. (2010) Hepatoprotective and antioxidant activity of ethanolic extracts of edible lotus (Nelumbo nucifera Gaertn.) leaves. Food Chem 120: 873-878.

43. Prakash J, Gupta S (2000) Chemopreventive activity of Ocimum sanctum seed oil. J Ethnopharmacol 72: 29-34.

44. Umar S, Golam Sarwar AH, Umar K, Ahmad N, Sajad M, et al. (2013) Piperine ameliorates oxidative stress, inflammation and histological outcome in collagen induced arthritis. Cell Immunol 284: 51-59.

45. Sunila ES, Kuttan G (2004) Immunomodulatory and antitumor activity of Piper longum Linn. and piperine. J Ethnopharmacol 90: 339-346.

46. Banerjee A, Dasgupta N, De B (2005) In vitro study of antioxidant activity of fruit. Food Chem 90: 727-733.
47. Dwivedi S (2007) Terminalia arjuna Wight \& Arn.--a useful drug for cardiovascular disorders. J Ethnopharmacol 114: 114-129.

48. Jadon A, Bhadauria M, Shukla S (2007) Protective effect of Terminalia belerica Roxb. and gallic acid against carbon tetrachloride induced damage in albino rats. J Ethnopharmacol 109: 214-218.

49. Rota MC, Herrera A, Martínez RM, Sotomayor JA, Jordán MJ (2008) Antimicrobial activity and chemical composition of Thymus vulgaris, Thymus zygis and Thymus hyemalis essential oils. Food Control 19: 681-687.

50. Orhan DD, Orhan N, Ergun E, Ergun F (2007) Hepatoprotective effect of Vitis vinifera $\mathrm{L}$. leaves on carbon tetrachloride-induced acute liver damage in rats. $J$ Ethnopharmacol 112: 145-151.

51. Pantoja CV, Chiang LC, Norris BC, Concha JB (1991) Diuretic, natriuretic and hypotensive effects produced by Allium sativum (garlic) in anaesthetized dogs. J Ethnopharmacol 31: 325-331.

52. Mascolo N, Jain R, Jain SC, Capasso F (1989) Ethnopharmacologic investigation of ginger (Zingiber officinale). J Ethnopharmacol 27: 129-140.

53. Abd el Nabi OM, Reisinger EC, Reinthaler FF, Still F, Eibel U, et al. (1992) Antimicrobial activity of Acacia nilotica (L.) Willd. ex Del. var. nilotica (Mimosaceae). J Ethnopharmacol 37: 77-79.

54. Davis L, Kuttan G (2000) Immunomodulatory activity of Withania somnifera. J Ethnopharmacol 71: 193-200.

55. Ray D, Thokchom I (2006) Antipyretic, antidiarrhoeal, hypoglycaemic and hepatoprotective activities of ethyl acetate extract of Acacia catechu Willd. in albino rats. Indian J Pharmacol 38: 408.

56. Nair PK, Melnick SJ, Wnuk SF, Rapp M, Escalon E, et al. (2009) Isolation and characterization of an anticancer catechol compound from Semecarpus anacardium. J Ethnopharmacol 122: 450-456.

57. Lansky EP, Newman RA (2007) Punica granatum (pomegranate) and its potential for prevention and treatment of inflammation and cancer. $J$ Ethnopharmacol 109: 177-206. 